\title{
Active Flow Separation Control on a High-Lift Wing-Body Configuration Part 1: Baseline Flow and Constant Blowing
}

\author{
Timo Kühn, Vlad Ciobaca* and Ralf Rudnik ${ }^{\dagger}$ \\ German Aerospace Center (DLR), 38108 Braunschweig, Germany \\ Burkhard Gölling ${ }^{\ddagger}$ \\ Airbus Operations GmbH, 28199 Bremen, Germany \\ Wiebke Breitenstein $\S$ \\ University of Applied Sciences Emden/Leer, 26793 Emden, Germany
}

\begin{abstract}
This paper describes the influence of grid resolution and turbulence modeling for a 3D transport aircraft in high lift configuration with massive flap separation. The flap is equipped with spanwise slotted active flow control (AFC) devices to allow studies on active separation control. The effects of constant slotted blowing on the high lift performance are highlighted. Oil flow pictures from a mid-scale experiment in the low speed wind tunnel of Airbus in Bremen (B-LSWT) serve as a validation database for the baseline CFD results. RANS calculations are carried out with and without constant blowing boundary conditions. The baseline flow is also investigated with a time-accurate URANS approach. One of the major outcomes of the AFC study is the demonstration of the feasibility to simulate AFC concepts on a 3D configuration. Constant blowing shows the beneficial effect that separation can largely be suppressed because of the energy added to the flow on the suction side of the flap. This study serves as a preceding validation for the subsequent pulsed blowing approach treated in Part 2.
\end{abstract}

\section{Nomenclature}

$\begin{array}{ll}\text { AFC } & =\text { Active Flow Control } \\ \text { B-LSWT } & =\text { Low Speed Wind Tunnel at Airbus in Bremen } \\ \text { CFD } & =\text { Computational Fluid Dynamics } \\ \text { CFL } & =\text { Courant Friedrichs Lewy [number] } \\ \text { DLR } & =\text { Deutsches Zentrum für Luft- und Raumfahrt [German Aerospace Center] } \\ \text { RANS } & =\text { Reynolds Averaged Navier Stokes [method] } \\ \text { SAO } & =\text { Spalart-Allmaras Original [turbulence model] } \\ \text { SST } & =\text { Shear Stress Transport [turbulence model] } \\ \text { URANS } & =\text { Unsteady Reynolds Averaged Navier Stokes [method] } \\ A_{r e f} & =\text { Wing Reference Area [m²] } \\ C_{D} & =\text { Drag Coefficient [-] } \\ C_{L} & =\text { Lift Coefficient [-] } \\ C_{\mu} & =\text { Blowing Momentum Coefficient [-] }\end{array}$

* Research Engineers, Institute of Aerodynamics and Flow Technology, Lilienthalplatz 7

${ }^{\dagger}$ Head of Transport Aircraft Branch, Institute of Aerodynamics and Flow Technology, Lilienthalplatz 7

¥Project Supervisor, High-Lift Devices Skill Group, Airbus-Allee 1

$\S$ Bachelor Student, Constantiaplatz 4 


$$
\begin{array}{ll}
m_{j} & =\text { Jet Exit Mass Flow }[\mathrm{kg} / \mathrm{s}] \\
N & =\text { Point Number [-] } \\
q_{\infty} & =\text { Farfield Stagnation Pressure }\left[\mathrm{kg} / \mathrm{m}^{3}\right] \\
r_{e f f} & =\text { Effective Refinement Ratio [-] } \\
v_{j} & =\text { Jet Exit velocity }[\mathrm{m} / \mathrm{s}] \\
y^{+} & =\text {Dimensionless First Grid Spacing [-] }
\end{array}
$$

\title{
I. Introduction
}

\begin{abstract}
IRBus Flight Physics, High Lift Devices, in Bremen and the DLR Institute of Aerodynamics and A Flow Technology in Braunschweig have conducted a collaborative pilot study on an industriallike three-element high-lift / wing-body configuration. The numerical studies are based on hybrid unstructured methods in use at DLR (TAU code and the commercial CENTAUR grid generation package). The code has been extended towards the capability to allow the design of wings with increased high lift performance enforced by active flow control (AFC) actuations.

Limited to two-dimensional (2D) and towards swept two-dimensional (2.5D) profile flows, such fluidic actuation systems were investigated experimentally within the project M-Fly ${ }^{1}$ of the German national aerospace program and numerically within the partner program M-Fly/AeroNext by the partners with the support of Airbus. ${ }^{2}$ Corresponding pilot investigations of AFC on an industriallike three-dimensional (3D) wing/body configuration have been carried out succesfully in the low speed wind tunnel of Airbus-Deutschland in Bremen (B-LSWT) using a 1:12.7 high-lift wing model with application of AFC on the high lift trailing edge device.

The present work describes first steps towards the simulation of continuous and later on pulsed actuated jet flows on the flap of this 3D high-lift wing-body configuration using the DLR TAU code. A prerequisite to simulate the benificial effect of AFC is the ability to consistently simulate the flow features of the baseline high-lift configuration without AFC, which is in the present case characterized by substantial portions of separated flow.

A main focus of the numerical investigations is to determine the influence of grid resolution in order to identify an appropriate numerical baseline set-up to capture the main flow features, while using a minimum grid size. In addition to the grid resolution study, three different turbulence models are used to assess the impact of physical modeling on the final solution.
\end{abstract}

\section{Grid Generation and Numerical Method}

The hybrid grids for the preliminary CFD investigation of the reference flow and also the actuated computations with AFC are generated with the commercial mesh generation software CENTAUR. ${ }^{3}$ For the approximation of the boundary layer, there are 25 prism layers with a target $y^{+}$-value of 1.0 ensuring an appropriate resolution of the viscous sublayer. Complicated areas with acute surface angles or small gaps are either treated with an automatic pullback of the first grid spacing or with minimal chopping of the prismatic advancing front. Both are methods to reduce the overall height of the prismatic grid. While the first method maintains the number of prism layers by locally lessening the prism height of every layer, the chopping leads to a reduction in the number of layers. There are only a few regions, where this problem arises, like the connection of the flap track fairings or the intersection of the aileron with the wing. The farfield distance is 100 reference chord lengths away from the geometry. For the grid refinement study, two additional meshes are derived starting from the coarsest grid level in order to get three subsequently refined mesh levels. The point number for the coarse, intermediate, and fine grid amounts to $17 \cdot 10^{6}, 25 \cdot 10^{6}$ and $37 \cdot 10^{6}$ points, respectively. A classical systematic grid refinement with a subsequent analytical error estimation is easily possible for fully structured grids, where a doubling or bisection of the points can be applied. ${ }^{4}$ With the available methods and tools, a similar approach is not practicable for unstructured meshes. Thus, the surface mesh refinement is limited to the flap system and the spoiler, because the work is focused on the flap separation region. An equal refinement in areas of lower interest would severely multiply the numerical effort limited by hardware constraints. For the same reason, the number of prism layers is kept constant and the refinement is focused on the 
surface elements. It is a possible way to refine unstructured meshes with the usage of an effective refinement ratio, which is for a three dimensional problem defined as:

$$
r_{\text {eff }}=\left(\frac{N_{\text {fine }}}{N_{\text {coarse }}}\right)^{\frac{1}{3}}
$$

This ratio is constant at 1.14 for the refinement from the coarse to the intermediate grid and from the intermediate to the fine grid. Figure 1 depicts the meshes used for the grid refinement study. For the active flow control studies, the grid is then extended in order to include the slots of the actuators. Primarily, the number of prism stacks was raised to 30 layers and the stretching ratio for the boundary layer prism extrusion was slightly increased. This procedure emerges to be very helpful in order to include deep slots on the flap surface with an automatic grid generation package as CENTAUR.

The flow solver used for the presented results is the finite volume compressible RANS solver TAU

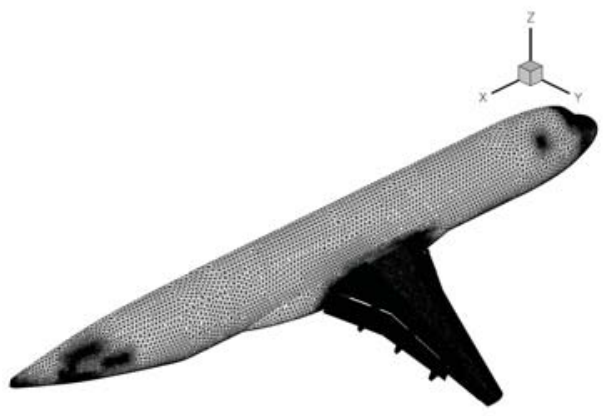

(a) Farfield view of the overall mesh

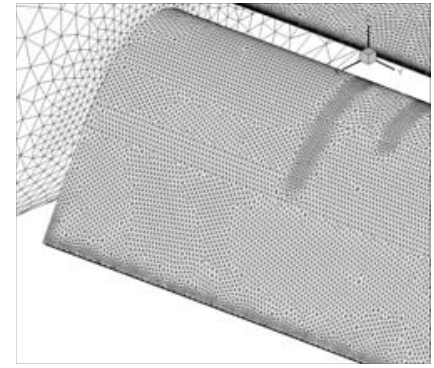

(b) Close view of the inboard flap edge (coarse mesh)

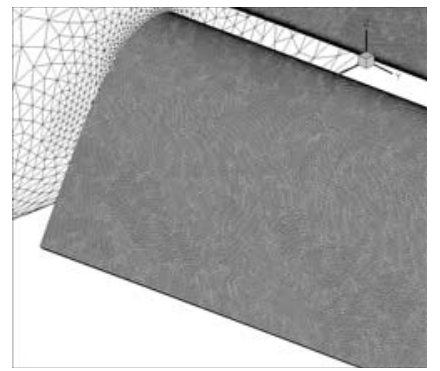

(c) Close view of the inboard flap edge (intermediate mesh)

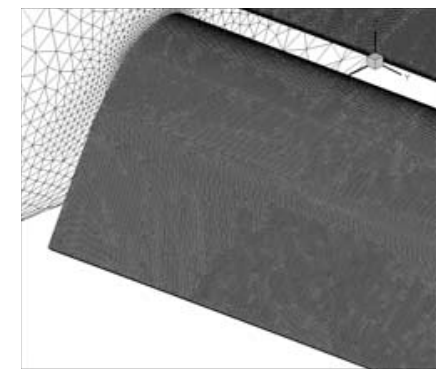

(d) Close view of the inboard flap edge (fine mesh)

Figure 1. Overview of the surface meshes

developed at DLR. ${ }^{5}$ A second order accurate central scheme with artificial dissipation is the method of choice for the discretization of the convective fluxes. The artificial dissipation based upon the settings of Jameson ${ }^{6}$ is applied with a $2^{\text {nd }}$ order dissipation term of $\frac{1}{2}$, and a $4^{\text {th }}$ order dissipation coefficient of $\frac{1}{64}$. The chosen approach for the time integration is a 3-stage Runge-Kutta time integration method using a CFL number of 1.2. In addition to a point explicit residual smoother, convergence is accelerated with a $3 \mathrm{~W}$-multigrid cycle. The URANS computations are carried out with a dual time stepping method and a physical time step size of $2.5 \cdot 10^{-5}$ seconds and 125 inner iterations. The choice of the time step size and the number of iterations is based on conclusions of part 2 of the paper, where the unsteady approach is examined in detail. Besides an investigation of the mesh influence, the comparison of different turbulence models is in the focus of this work. On the one hand, the 1-equation turbulence model of Spalart and Allmaras ${ }^{7}$ in its original version (SAO) is used, and on the other hand the 2-equation k- $\omega$-type models of Wilcox ${ }^{8}$ and the advanced Shear Stress Transport model (SST) from Menter. ${ }^{9}$ For the two 2 -equation models a different wall limiter type for the $\omega$-equation is used, where the lower bound of $\omega$ is limited based on experimental 


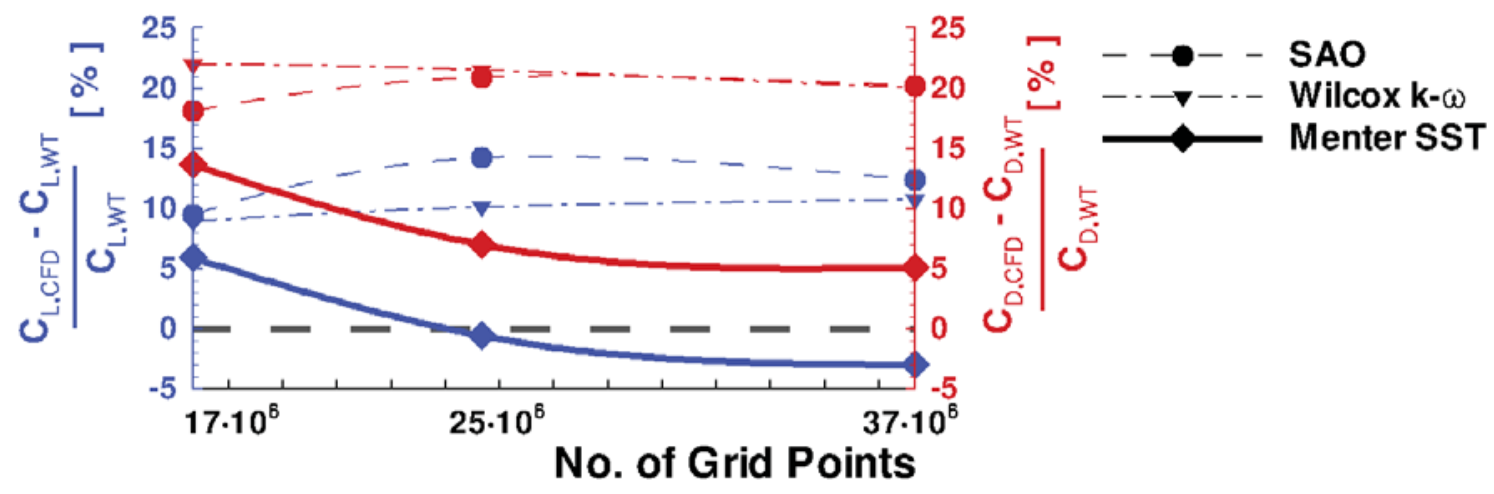

Figure 2. Grid influence on the overall lift and drag cofficient in percentages of the wind tunnel measurements

assumptions for the wall roughness. ${ }^{10}$ This limiter previously showed good and stable results for cases with high complexity. On the constant blowing boundary condition, the velocity on the outlet plane is set to a fixed value of $150 \mathrm{~m} / \mathrm{s}$. These are typical working conditions of the active flow control actuators. This value corresponds to a actuator/freestream velocity ratio of approximately 2.0, which is needed at higher flap deflection angles. ${ }^{11}$ In order to relate to the wind tunnel tests, the setup is determined with the Mach number, the Reynolds number, the corresponding reference length of the model and the farfield temperature. The Reynolds number of the wind tunnel experiment in Airbus' low speed wind tunnel in Bremen (B-LSWT) is $1.5 \cdot 10^{6}$ and the Mach number is 0.2 , whereas the farfield temperature is $300 \mathrm{~K}$. Based on the results of the wind tunnel campaign, an angle of attack of $\alpha=7^{\circ}$ in the linear regime of the lift curve has been selected for the CFD analysis. For this angle of attack, the wind tunnel database was very comprehensive.

\section{Results}

The wind tunnel experiments for the baseline flow reveal a strong separation on the inboard and outboard flap. The discussion of the overall numerical results can be divided into three major parts: 1 . the investigation of the baseline flow with its implications for the usage of the necessary grid resolution and the turbulence model, 2. a study on the introduction of slotted actuators and their influence on the aerodynamics, and 3. the subsequent analysis of the impact of active flow control.

\section{III.A. Baseline Configuration}

First of all, the influence of the mesh should be highlighted by evaluating the force coefficients (in relation to the wind tunnel measurements) for the three grids. As illustrated in figure 2, the trends of the grid refinement study are not consistent for some of the values. The most prominent anomaly is visible for the SAO results where the evolution of the coefficients is not monotonic. Both the lift and the drag coefficient first increase and then decrease with further refinement. But there is also a discrepancy in the behaviour of the two 2-equation-model results. Despite the fact, that here the trends are monotonic for both the lift and the drag coefficient, the models show an inversion of the direction. For the Wilcox model, the lift coefficient increases steadily, while the SST model results in a steady decrease. In turn, the drag coefficient results in a steady decrease for both the Wilcox and the SST model. But in conclusion, only the unclear trend of the SAO models is suspicious, because grid convergence is characterized by an asymptotic behaviour of a representative variable, but not by the direction of this trend. Altogether, only the two-equation models produce the specific asymptotic progression of a grid convergence study. It could be criticized, that the refinement was done only locally. But recent studies show that for complex CFD studies even a very accurate refinement study with genuine grid families does not guarantee a classical grid convergence behaviour with force coefficients in the asymptotic range. ${ }^{12,13}$ In the present study, wind tunnel data is available so that the meshes can additionally be judged by a comparison with the experimental data. 
Table 1. Deviation of the computed force coefficients compared to the measured data

\begin{tabular}{|c|c|c|c|c|c|c|c|c|c|}
\cline { 2 - 10 } \multicolumn{1}{c|}{} & \multicolumn{4}{c|}{ coarse } & \multicolumn{3}{c|}{ intermediate } & \multicolumn{3}{c|}{ fine } \\
\cline { 2 - 10 } & SAO & Wilcox & SST & SAO & Wilcox & SST & SAO & Wilcox & SST \\
\hline$\frac{C_{L, C F D}-C_{L, W T}}{C_{L, W T}}[\%]$ & 9.5 & 8.9 & 5.9 & 14.2 & 10.1 & -0.6 & 12.4 & 10.7 & -3.0 \\
\hline$\frac{C_{D, C F D}-C_{D, W T}}{C_{D, W T}}[\%]$ & 18.1 & 22.0 & 13.6 & 20.9 & 21.5 & 7.0 & 20.2 & 20.0 & 5.1 \\
\hline
\end{tabular}

Besides the evolution of the trends, also the comparison of the force coefficient magnitudes listed in table 1 shows an unambigous indication towards the Menter SST computations with the best match in lift for the intermediate mesh. Keeping in mind that computational drag prediction is an ongoing matter of numerous research activities, ${ }^{14}$ one should focus on the lift coefficient while comparing absolute values. Nevertheless, comparing the turbulence models, the best drag agreement can also be found for the SST calculations. Concerning the magnitudes of the offset from the numerical results to the experimental data, they scatter within a large interval, and only for the SST model and the fine and intermediate grid this deviation is acceptable. The results range from over $20 \%$ to $-3 \%$, which is not surprising because of the large separation region that strongly contributes to the lift and drag of the model. A final concluding remark regarding the global lift and drag coefficient analysis stated here is mandatory. As noted in the last chapter, there are always uncertainties concerning the validity of mesh resolution studies with unstructured grids, especially when only local refinements are addressed. This must be kept in mind evaluating the findings above.

Now attention should be paid to the grid influence on the flow topology. Taking a look at the friction lines of the three successively refined meshes in figure 3, there is one striking peculiarity. Independent of the type of turbulence model, the coarse mesh approach triggers an edge vortex at the inner edge of the inboard flap that is grid induced, because an inrease of the point number results in a complete disappearance of this vortex. It is much more distinct for the SAO and Wilcox k- $\omega$ results but also visible for the SST. A further refinement from the intermediate to the fine grid reveals only marginal differences in the overall flow behaviour so that this comparison along with the findings above justifies the usage of an intermediate mesh approach. Altogether, the grid refinement gave good evidence for a sufficient mesh resolution and serves as a calibration for further CFD work incorporating this model.

Next, the influence of the turbulence model on the flow topology will be investigated. The impact of the turbulence models can be observed again in figure 3, where the friction lines of the CFD results are depicted in comparison with oil flow pictures of the wind tunnel experiment. While the outboard separation is simulated by all turbulence models to a certain extent, the inboard separation is only reproducable by the Menter SST-model. In figures 3(h) to 3(j) the structure of the flow above the flap upper surface shows a reverse flow region close to the inboard flap track fairing, sometimes referred to as "owls eyes". This is in good accordance with the flow visualisation of the experiments, although there is a small deviation from the measured separation line. In contrast to that, the remaining turbulence models do not forecast a comparable inboard separation (figures $3(\mathrm{~b})$ to $3(\mathrm{~g})$ ). As regards these two inferior models, only small advantages (i.e. in this case a slightly better separation and lift prediction compared to the wind tunnel data, see again table 1) elevate the Wilcox over the SAO model. These findings coincide with the recommendation towards the Menter SST model for strongly separated flows where adverse pressure gradients are present. ${ }^{15}$ Neglecting the prevailing inconsistency of the drag coefficients with the uncertainties attached to numerical drag prediction, a ranking of the three turbulence models becomes apparent. The Menter SST model clearly stands out being the only model succesfully forecasting the large inboard separation. It is followed by the Wilcox two equation model and the SAO model. These two show differences in the flow topology and large offsets in comparison with the measured force coefficients. Regarding the grid fineness, the results obtained with the intermediate grid yield the best compromise between quality and efficiency.

In anticipation of the studies in part 2 of the paper, the baseline configuration was also computed on the intermediate grid with an unsteady URANS approach in order to see whether the stationary results remain unaffected. The overall physical time of over 0.18 seconds allows a perturbation to propagate from its origin on the geometry for more than 100 flap reference length units through 


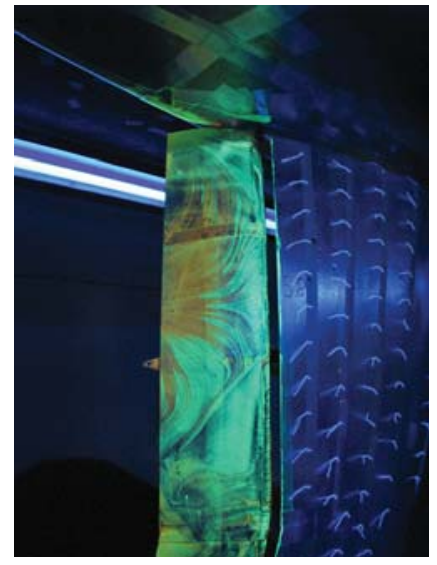

(a) Oil flow picture of the wind tunnel experiment

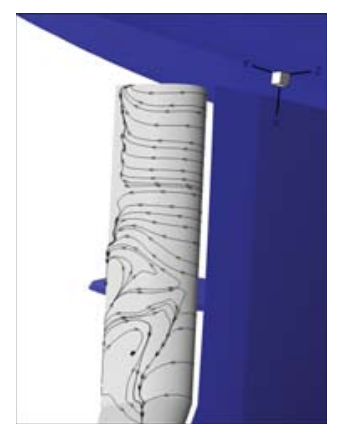

(c) Numerical results of the SAO computations (intermediate mesh)

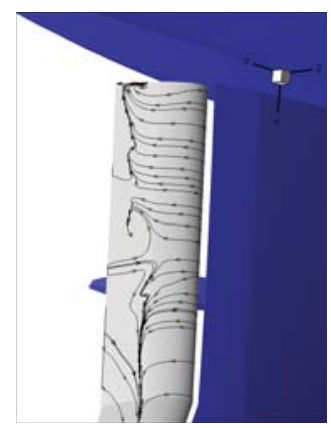

(f) Numerical results of the Wilcox k- $\omega$ computations (intermediate mesh)

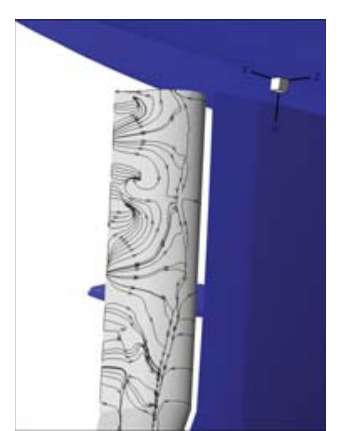

(i) Numerical results of the Menter SST computations (intermediate mesh)

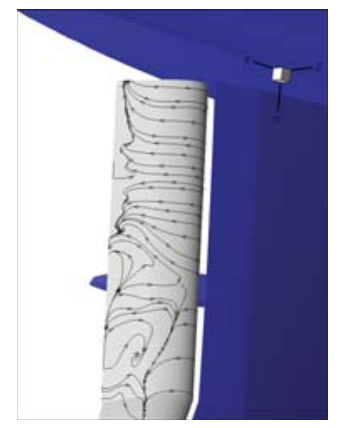

(d) Numerical results of the SAO computations (fine mesh)

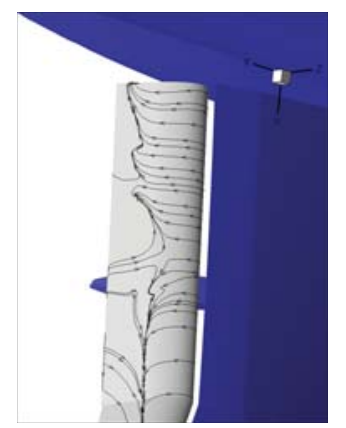

(g) Numerical results of the Wilcox k- $\omega$ computations (fine mesh)

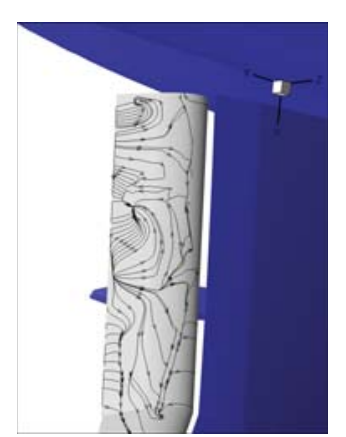

(j) Numerical results of the Menter SST computations (fine mesh)

(h) Numerical results of the Menter SST computations (coarse mesh)

Figure 3. Influence of the grid refinement in direct comparison with wind tunnel experiments for the baseline flow

$$
6 \text { of } 11
$$




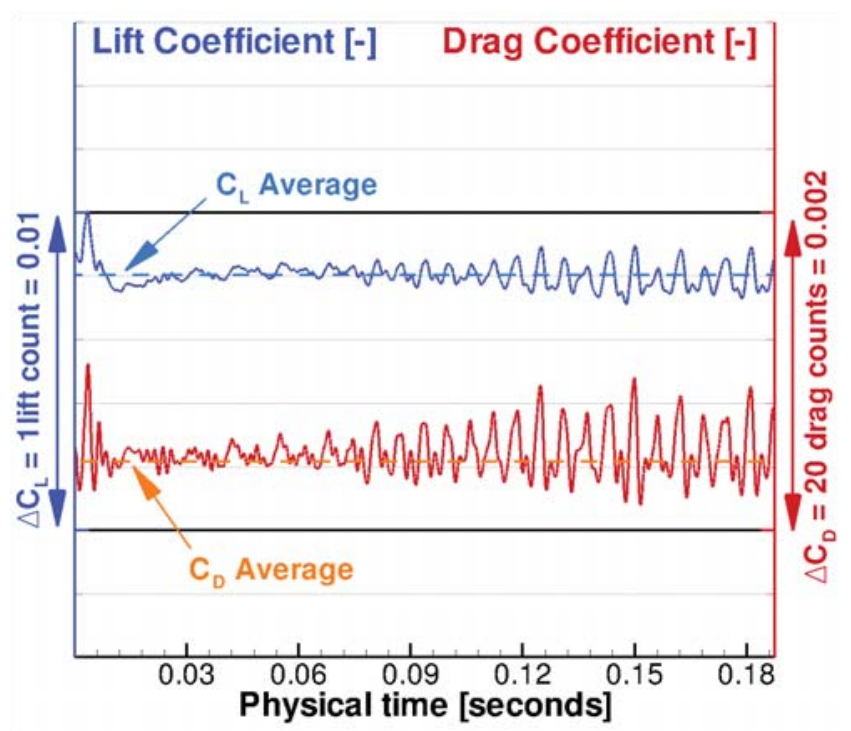

Figure 4. Force coefficients of an unsteady URANS computation for the baseline flow

the flow field. Figure 4 shows the progression of the lift and drag coefficient along with its corresponding average values for this unsteady computation. Because this computation was restarted from the steady state solution, the reference point of the stationary results is situated at $t=0 \mathrm{~s}$. One observation is that after a short transition period of approximately $0.03 \mathrm{~s}$, the solution starts oscillating around the average value that is very close to the reference value. The oscillation is not strictly harmonic but follows a recurring pattern. Longer computation time does not change this behaviour any more. With respect to the scale of the axes $\left(\Delta C_{L}=0.01, \Delta C_{D}=0.002\right)$, both the size of the oscillation and the offset from the initial result is neglectable. With this information the unsteady results complete the study of the baseline model.

\section{III.B. Slot Integration}

In the previous chapter, guidelines were derived for the choice of the turbulence model and the grid resolution of the baseline geometry. In the following, this strategy is adapted for all computations incorporating active flow control devices. For the constant and the pulsed blowing via active flow control elements, the geometry is extended with 21 separate slots on the suction side of the inboard and outboard flap. There are 6 and 15 slots placed on the inboard and outboard side, respectively. They are separately resolved with cells as depicted in figure 5 meaning that the interior of every flow control actuator slot is filled with grid elements. The gaps near the fairings are due to the assembly limits of the flap track connectors inside the flap of the wind tunnel model. Of course,

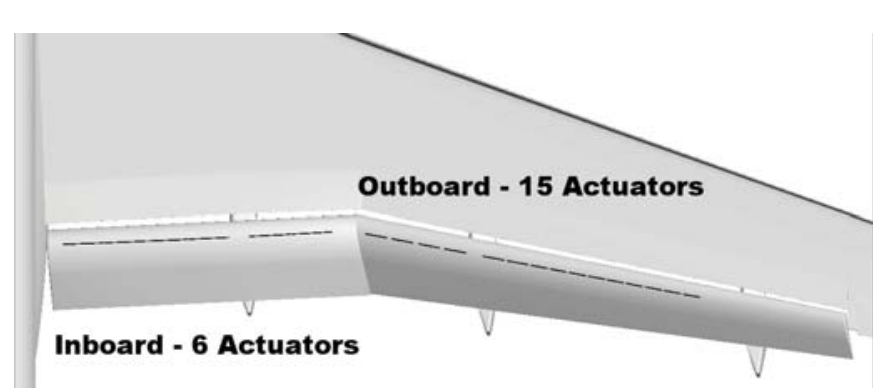

(a) Placement of the actuator slots on inboard and outboard flap

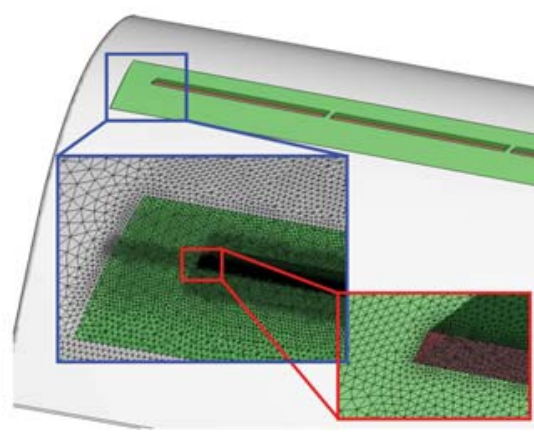

(b) Slot surface meshing strategy

Figure 5. Positioning and meshing strategy in the vicinity of the slot actuators 
the introduction of active flow control slots has an impact on the overall mesh topology. In the previous section it was already mentioned that the number of prism cells was increased. This leads to a higher number of points of $30 \cdot 10^{6}$. Introducing very small cells on the flap surface through the slot panels also leads to an irregular shape of the outer hull of the prism stacks. This behaviour is difficult to avoid because of the strong automation of the grid generation process.

The analysis shows that the lift coefficient is $6 \%$ smaller compared to the results of the baseline model. The main reason is the reduction of the flap suction peak. It is not self-evident that this is solely a consequence of the slot integration. The deformation of the prism structure might as well contribute to the lift loss. Figure 6 reveals the differences between the pressure distribution with and without slots. Most striking is the strongly reduced suction peak in the kink area for the case with slots. Provoked by the leading edge sweep of the outboard flap, high lift configurations with an inboard (no sweep) and outboard flap (sweep) generally feature an outward motion of the fluid in the kink area. By introducing the slots (especially the two slots close to the kink), this motion is suppressed leading to a reduced ability for a high flow acceleration. In second consequence, the reduction of the flap circulation also affects the main wing to a certain extent up to approximately $30 \%$ upstream of the main wing trailing edge. The effect is very small and difficult to visualize with any colour mapping. However, the colouring of the spoiler hinge line acceptably reveals this lift loss of the main wing for the case with slots (fig. 6(b)). This so-called circulation effect is typical for multi-element high lift aerodynamics as first described by A.M.O. Smith. ${ }^{16}$ The theorem states that an increased circulation of the downstream element causes the trailing edge of the adjacent upstream element to be in a region of high velocity. For the case with slots, the reduction of the flap circulation due to the installation of the slots leads to the opposite occurence that the wing trailing edge pressure is reduced. However, this effect does not stretch out to the main wing leading edge, where the differences tend to zero.

The key point of this study is that the general flow topology persists, which is of high importance for the following work. Separation is a little bit shifted to the flap leading edge. But for the investigation of active flow control, a massively separated flap is desired, anyway. It was shown that this was already the case for the baseline geometry and that an incorporation of the slots promotes the flap separation to a minor degree.

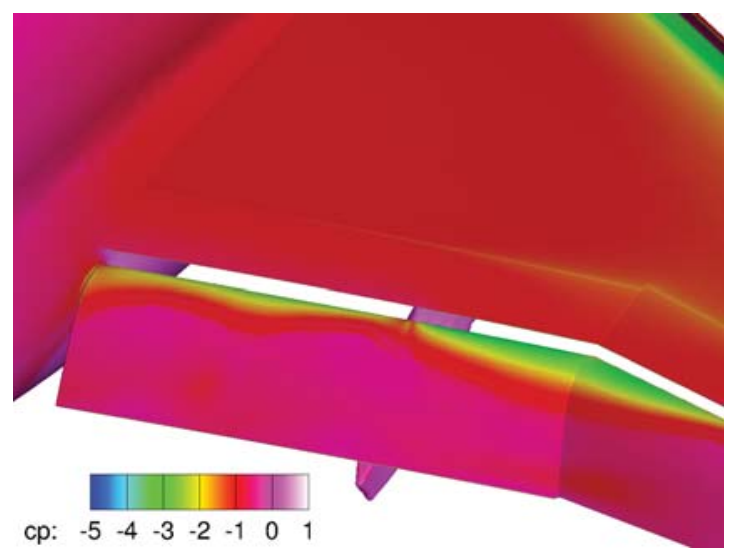

(a) Without slots

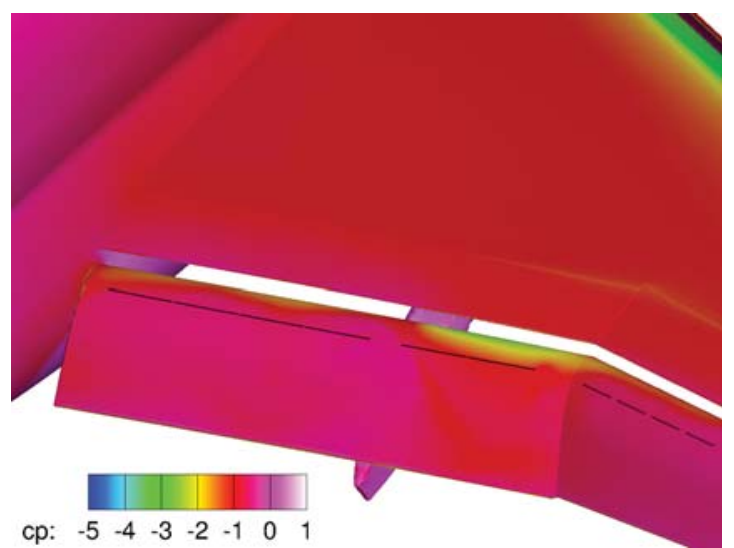

(b) With slots

Figure 6. Impact of the slot integration on the pressure distribution for the case without AFC

\section{III.C. Constant Blowing}

Refering to the baseline flow, the physical reason for the separation on the suction side of the flap is the strong adverse pressure gradient that the flow can not withstand due to a lack of dynamic energy. The control flow through the slotted actuators leads to a reenergization of the boundary layer and therefore enforces the ability to withstand separation tendencies. Its momentum is defined by the blowing momentum coefficient $C_{\mu}$ :

$$
C_{\mu}=\frac{m_{j} \cdot v_{j}}{q_{\infty} \cdot A_{r e f}}
$$


The definition contains the jet mass flow $m_{j}$ through the actuator slots with the jet velocity $v_{j}$, the farfield stagnation pressure $q_{\infty}$, and the wing reference area $A_{\text {ref }}$ that is also used for all aircraft based force coefficients. For constant blowing, the jet velocity and mass flow is constant, in this case with a value of $C_{\mu}=0.5 \%$.

Flow separation or its suppression can be visualized by the flow signature on the surface. The signatures on the surface, as they are depicted in figures $7(\mathrm{a})$ and $7(\mathrm{c})$, show the effect of the separation suppression. One can see, that the originally completely separated flow above the flap in figure $7(\mathrm{a})$ is substituted by a mostly attached flow. Remember, that some slots are divided by larger gaps in regions where the flap does not bear enough space for the actuation hardware. Close to these gaps, noticeable portions of separation still exist (e.g. in the kink area). At the outer end of the outboard flap near the third flap track fairing, the slots are completely missing which results in an almost unconstraint separation as without AFC. It is important to note, that constant blowing evidently improves the performance of the high lift device. This is clearly visible in the refered figures where also the surface pressure distribution is shown. The avoidance of flow separation is followed by a strong increase of the suction peak. This is also reflected in the overall lift coefficient that is enhanced by 0.24 .

Naturally, not only the signature on the surface is an indicator for the differences between the case with and without AFC. Most of all, it is a 3 dimensional phenomenon that is in first consequence visible in the field. Figures 7(b) and 7(d) show a slice through the wake region behind the inboard flap. Without the application of active flow control this wake is mainly characterized by a continous dead water region from the inboard edge all over the flap span. In contrast to that, constant blowing leads to a destruction of this continuous structure but leaves some remaining distinct vortices that are caused by the unsuppressable leftovers of the large separation area, namely at the inward junction of the wing and the belly fairing, above the flap track fairings, and in the kink area between inboard and outboard flap.

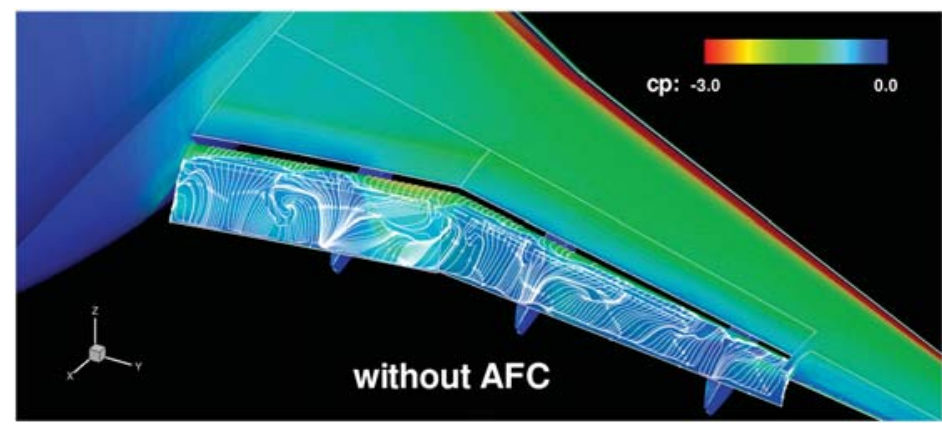

(a) Friction lines and pressure distribution of the inboard and outboard flap without constant blowing active flow control

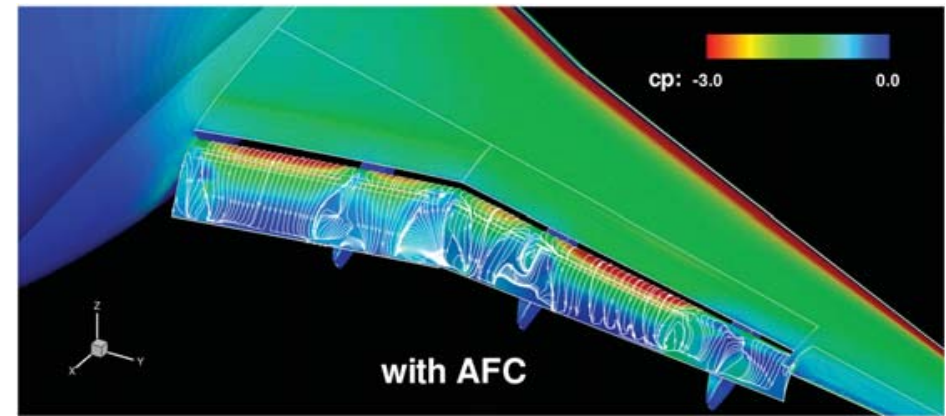

(c) Friction lines and pressure distribution of the inboard and outboard flap with constant blowing active flow control

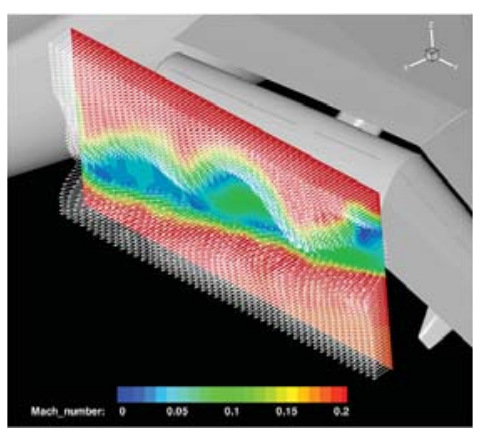

(b) Mach contours of the inboard flap wake region without constant blowing active flow control

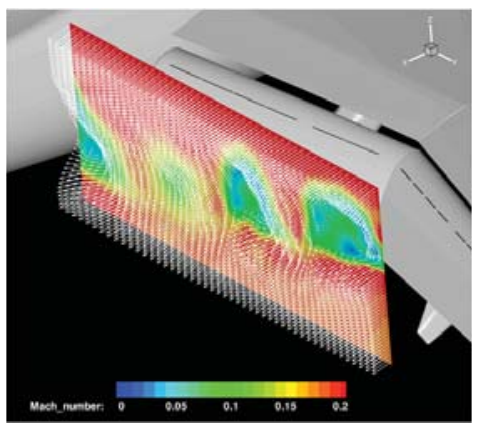

(d) Mach contours of the inboard flap wake region with active flow control

Figure 7. Flow topology in the vicinity of the aircraft flap system with and without constant blowing active flow control 


\section{Conclusion}

The present work contains a numerical investigation of a high-lift wing-body configuration with a separated flow above the inboard and outboard flap. The baseline flow is computed with three different turbulence models and three succesively refined grids and compared with wind tunnel data. Afterwards, the influence of active flow control slots on the baseline flow is described. Finally, the impact of constant blowing on the high lift performance is highlighted.

An important aspect of the considered flow around the high lift model was the full-span separation on the trailing edge high lift system. This feature of the baseline model was predicted accurately only with the Menter SST model in combination with an intermediate or fine grid. The intermediate mesh in combination with the SST model gives the best compromise between quality and computational turn-around times. URANS computations were carried out to create a link between the baseline flow in part 1 and the pulsed blowing application in part 2 of the paper where a time-accurate treatment of the flow is necessary. The URANS computations affirm that both approaches result in the same flow behaviour and a good match of the force coefficients compared to the steady approach. An incorporation of the slots for the actuation has only small effects on the overall flow topology but is accompanied by an even stronger suppression of the flap suction peak and a lift loss. The application of constant blowing prevents a complete detachment of the suction side flow and leads to an increase in high lift performance. The principal feasibility of applying active flow control elements on the complex high lift geometry under consideration could be shown. Part 2 of the paper ${ }^{17}$ deals with the numerical investigation of the more efficient pulsed blowing approach including a final comparison with AFC wind tunnel data. ${ }^{18}$

\section{Acknowledgments}

These investigations by DLR were supported by Airbus in the frame of a German national funded R\&T project. The authors would like to especially thank the project executing organization, the high lift devices skills group and the methods and tools group at Airbus in Bremen for their support and the clearance of the present content.

\section{References}

${ }^{1}$ Wild, J., Wichmann, G., Haucke, F., Peltzer, I., and Scholz, P., "Large Scale Separation Flow Control Experiments within the German Flow Control Network," AIAA Paper 2009-530, January 2009.

${ }^{2}$ Togiti, V., Ciobaca, V., Eisfeld, B., and Knopp, T., "Numerical Simulation of Steady Blowing Active Flow Control Using a Differential Reynolds Stress Model," KATnet II Conference on Key Aerodynamic Technologies, Bremen, Germany, May 2009.

${ }^{3}$ http://centaursoft.com, May 2010.

${ }^{4}$ Roache, P., Fundamentals of Computational Fluid Dynamics, Hermosa Publishers, Albuquerque, New Mexico, 1998, Chap. 19.

${ }^{5}$ Gerhold, T., "Overview of the Hybrid RANS Code TAU," MEGAFLOW - Numerical Flow Simulation for Aircraft Design, Vol. 89 of Notes on Numerical Fluid Mechanics and Multidisciplinary Design, Springer Verlag, 2005, pp. 81-92.

${ }^{6}$ Jameson, A., Schmidt, W., and Turkel, E., "Numerical Solutions of the Euler Equations by Finite Volume Methods Using Runge-Kutta Time-Stepping Schemes," AIAA Paper 81-1259, June 1981.

${ }^{7}$ Spalart, P. and Allmaras, S., "A One-Equation Turbulence Model for Aerodynamic Flows," AIAA Paper 92-0439, Jan. 1992.

${ }^{8}$ Wilcox, D., "Reassessment of the Scale-Determining Equation for Advanced Turbulence Models," AIAA Journal, Vol. 26, No. 11, 1988, pp. 1299-1310.

${ }^{9}$ Menter, F., "Two-Equation Eddy-Viscosity Turbulence Models for Engineering Applications," AIAA Journal, Vol. 32, No. 8, 1994, pp. 1598-1605.

${ }^{10}$ Rudnik, R., "Evaluation of the Performance of Two-Equation Turbulence Models for Airfoil Flows," Ph.D. Dissertation, Transportation and Applied Mechanics Dept., Technical University Berlin, Germany, 1997.

${ }^{11}$ Nagib, H., Kiedaisch, J., Reinhard, P., and Demanett, B., "Active Flow Control for High Lift Airfoils: Separation versus Circulation Control," 45th AIAA Aerospace Sciences Meeting, Reno, Nevada, January 2011.

${ }^{12}$ Rumsey, C., Long, M., Stuever, R., and Wayman, T., "Summary of the First AIAA High Lift Prediction Workshop (invited)," 49th AIAA Aerospace Sciences Meeting, Orlando, Florida, January 2011.

${ }^{13}$ Crippa, S., Melber-Wilkending, S., and Rudnik, R., "DLR Contribution to the First High Lift Prediction Workshop," 49th AIAA Aerospace Sciences Meeting, Orlando, Florida, January 2011.

${ }^{14}$ Vassberg, J., Tinoco, E., Mani, M., Brodersen, O., Eisfeld, B., Wahls, R., Morrison, J., Zickuhr, T., Laflin, 
K., and Mavriplis, D., "Abridged Summary of the Third AIAA Computational Fluid Dynamics Drag Prediction Workshop," AIAA Journal of Aircraft, Vol. 45, No. 3, pp. 781-798, 2008.

${ }^{15}$ Menter, F., Kuntz, M., and Langtry, R., "Ten Years of Industrial Experience with the SST Turbulence Model," Turbulence, Heat and Mass Transfer 4, 2003, pp. 625-632.

${ }^{16}$ Smith, A., "High-Lift Aerodynamics," AIAA 6th Aircraft Design, Flight Test and Operations Meeting, 1974.

${ }^{17}$ Ciobaca, V., Kühn, T., Rudnik, R., Bauer, M., and Gölling, B., "Active Flow Separation Control on a HighLift Wing-Body Configuration - Part 2: The Pulsed Blowing Application," will be presented at the AIAA Applied Aerodynamics Conference 2011, Honolulu, Hawaii.

${ }^{18}$ Gölling, B., "High Lift Active Flow Control Investigations in National R\&T Projects," KATnet II Conference on Key Aerodynamics Technology, Bremen, Germany, May 2009. 